\title{
No Genotoxicity Is Detectable for Escherichia coli Strain Nissle 1917 by Standard In Vitro and In Vivo Tests
}

\author{
Silke Dubbert ${ }^{1}$, Birgit Klinkert ${ }^{1}$, Michael Schimiczek ${ }^{1}$, Trudy M. Wassenaar ${ }^{2}$ and Rudolf von Bünau ${ }^{{ }^{*}}$ \\ ${ }^{1}$ Ardeypharm GmbH, Loerfeldstraße 20, 58313 Herdecke, Germany \\ ${ }^{2}$ Molecular Microbiology and Genomics Consultants, Tannenstraße 7, 55576 Zotzenheim Germany
}

Received: 09 Dec 2019; accepted: 17 Feb 2020

\begin{abstract}
Probiotic Escherichia coli strain Nissle 1917 (EcN) has a long history of safe use. However, the recently discovered presence of a pks locus in its genome presumably producing colibactin has questioned its safety, as colibactin has been implicated in genotoxicity. Here, we assess the genotoxic potential of EcN. Metabolic products were tested in vitro by the Ames test, a mutagenicity assay developed to detect point mutation-inducing activity. Live EcN were tested by an adapted Ames test. Neither the standard nor the adapted Ames test resulted in increased numbers of revertant colonies, indicating that $\mathrm{EcN}$ metabolites or viable cells lacked mutagenic activity. The in vivo Mammalian Alkaline Comet Assay (the gold standard for detecting DNA-strand breaks) was used to determine potentially induced DNA-strand breaks in cells of the gastro-intestinal tract of rats orally administered with viable EcN. Bacteria were given at $10^{9}-10^{11}$ colony forming units (CFU) per animal by oral gavage on 2 consecutive days and daily for a period of 28 days to 5 rats per group. No significant differences compared to negative controls were found. These results demonstrate that $\mathrm{EcN}$ does not induce DNA-strand breaks and does not have any detectable genotoxic potential in the test animals.
\end{abstract}

Keywords: genotoxicity, probiotic, E. coli Nissle, colibactin, Ames test, comet assay

\section{Introduction}

Members of the species Escherichia coli can be either commensal to humans or pathogenic to the host, depending on the strain in question. The commensal nature of some $E$. coli strains have resulted in their use as probiotics, with the classical example of E. coli strain Nissle 1917 (EcN), which has been used as a probiotic for more than 100 years [1]. However, despite this long-term use in the absence of obvious side effects, its safety could be questioned, as the strain contains the polyketide synthesis ( $p k s$ ) locus that is responsible for production and secretion of colibactin [2]. The pks locus is approximately $50-\mathrm{kb}$ long and typically contains 16 genes in the same orientation $(c l b B-c l b Q$, sometimes interrupted by the presence of other genes), with the regulatory genes $c l b A$ and $c l b R$ in the opposite orientation (Figure 1). The pks genes encode 3 polyketide synthases (ClbC, ClbI, and $\mathrm{ClbO}), 3$ non-ribosomal peptide synthases $(\mathrm{ClbH}, \mathrm{ClbJ}$, and $\mathrm{ClbN}$ ), and 2 proteins with dual functions of both $\mathrm{ClbB}$ and $\mathrm{ClbK}$ and accessory proteins. In combination, these gene products produce, activate (e.g., peptidase $\mathrm{ClbP}$ ), and secrete (transporter $\mathrm{ClbM}$ ) the complex molecule colibactin. Colibactin contains a highly reactive cyclopropane group. The product of gene $c l b R$ regulates the transcription of $c l b A$, whose product $\mathrm{ClbA}$ again activates at least 2 promotors to produce polycistronic messengers [2, 3]. All genes except for clbM are required to actively express colibactin $[2,3]$. However, colibactin has not been purified and its structure is only inferred from precursor forms.

*Author for correspondence: Ardeypharm GmbH, Loerfeldstrasse 20, 58313 Herdecke; E-mail: vbuenau@ardeypharm.de.
Colibactin has been implicated in genotoxicity and may be involved in the onset of colorectal cancer [3-7]. In vitro and in vivo models have demonstrated that pks-containing $E$. coli strains are able to introduce DNA damage in exposed eukaryotic cells [3-9]. By means of knock-out mutants, it was demonstrated that the pks locus is responsible for the presumed genotoxicity [3], and heterologous expression of the locus corroborated these findings [10].

The impairment by genotoxic compounds is based on induction of mutations or DNA damage to subjected cells. Induced mutations can revert existing substitutions, a possibility that is assessed by the Ames test [11]. For this test, a potential mutagenic agent is incubated with specific E. coli or Salmonella reporter mutant strains that are auxotrophic for histidine or tryptophan. If the agent induces mutations, the auxotrophy is repaired by a reverse mutation, and revertant colonies can grow on minimal agar plates lacking this amino acid. The number of revertant colonies is a measurement of the genotoxic activity of the agent, and the Ames test was shown to be a suitable test to screen mutagenicity [12]. However, since the test depends on the growth of E. coli or Salmonella reporter strains, potential mutagenicity of a living bacterial strain cannot be determined, as it would overgrow the reporter strain, unless the test is modified.

DNA strand breaks induced by genotoxic agents can be visualized by the so-called Comet assay, developed in 1984 [13] and modified 4 years later in order to detect single-strand DNA breaks, too [14]. Recommendations for the Comet assay were expressed in 2003 [15] and in 2012 [16]. The Ames test and the Comet assay are both implemented by the Organization for Economic Co-operation and Development (OECD) to detect genotoxicity of a given compound $[17,18]$.

This is an open-access article distributed under the terms of the Creative Commons Attribution-NonCommercial 4.0 International License (https://creativecommons.org/licenses/by-nc/4.0/), which permits unrestricted use, distribution, and reproduction in any medium for non-commercial purposes, provided the original author and source are credited, a link to the CC License is provided, and changes - if any - are indicated. 


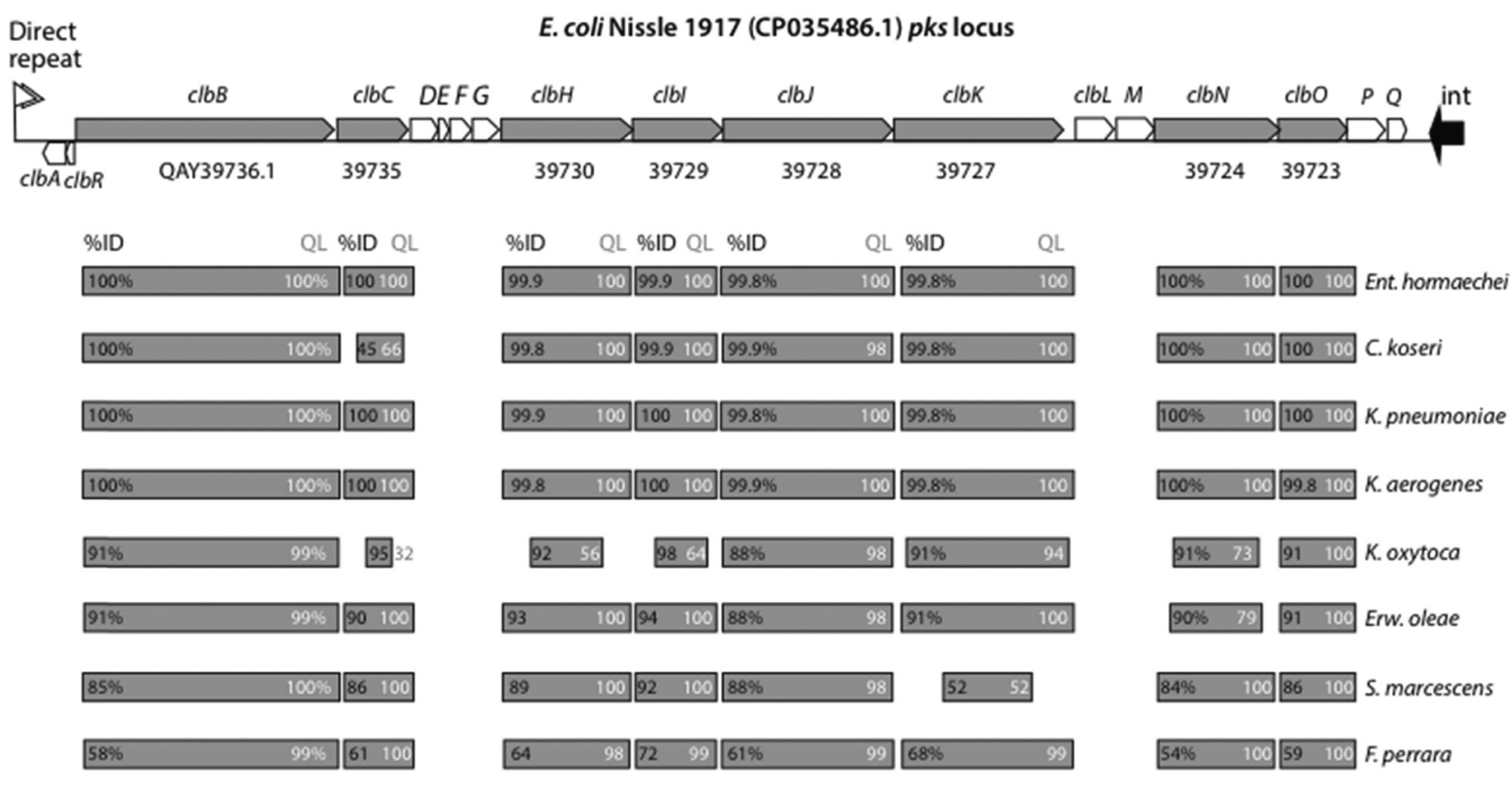

Figure 1. Degree of conservation of $p k s$ genes among Gram-negative bacteria. The top drawing shows the pks locus of EcN (genome accession number CP035486.1), with accession numbers of the polyketide synthases and non-ribosomal peptide synthases (grey gene arrows) shown below the analyzed genes. The amino acid sequences of these genes were used as query in BlastP. The blocks below the locus show the reported top scores per species of other Gram-negative bacteria, with percentage identity (\%ID, values in black) and percentage of query length coverage (\%QL, values in white). Hits to an unspecified Salmonella species (strain HMSC13B08) were ignored. Since the accessory genes (shown in white) may share functional domains with genes not related to the pks locus, their conservation was not assessed

Although in vitro models that are independent of test animals can be used for primary screening of genotoxicity, in vivo tests should be performed to confirm any measured effect, for which the Comet assay is a cheap, rapid, and sensitive method [19]. In this assay, rats are exposed to a test compound for a given period of time, after which the animals are sacrificed, and the target tissue is collected. Isolated cells from this tissue are embedded in a thin layer of agarose, lysed under high salt concentrations, and the DNA is allowed to unwind and denature using alkaline conditions. The liberated DNA is subjected to agarose gel electrophoresis, allowing any DNA fragments (indicative of damaged DNA) to migrate faster than non-damaged DNA molecules. This produces a comet-shaped pattern, after which the test is named. The relative intensity of the visible 'tail' depends on the frequency of DNA breaks, and this can be assessed using computer-based optical analysis. The intensity of the comet tail relative to the total intensity (head plus tail) reflects the amount of DNA breakage $[20,21]$. The Comet assay is recommended to be performed in rats, and the genotoxic potential of the test substance should be assayed in the target tissue(s) with respect to the most likely route of exposure [18].

Genotoxic effects of colibactin produced by various $E$. coli strains have been demonstrated in a number of murine models, which are summarized elsewhere, but all have their limitations [22]. Since rats are more easily colonized by E. coli than mice, and since rats are the standard test animal for genotoxic testing in combination with the Comet Assay, we chose that model to test any genotoxic activity of $\mathrm{EcN}$ in vivo.

The aims of this study were therefore to assess any mutagenic or genotoxic properties of $\mathrm{EcN}$ by (1) in vitro testing of spent supernatant in the classical Ames test; (2) in vitro testing of viable EcN using an adapted Ames test; and (3) in vivo testing using rats exposed to $\mathrm{EcN}$ for 2 time periods followed by the Comet Assay performed on the intestinal tissue in accordance to international guidelines. Before reporting the experimental results, the conservation of $8 \mathrm{pks}$ genes in other species is briefly summarized.

\section{Materials and Methods}

Assessment of Conservation of Pks Genes in Bacterial Species. Eight genes of the pks locus from the E. coli Nissle 1917 genome sequence (National Center for Biotechnology Information [NCBI] accession number CP035486.1) coding for polyketide synthases, non-ribosomal peptide synthases, or both, were used as query in quick-BlastP at NCBI with default settings except for the output, which was increased to 500 hits. The highest percentage of query length (\%QL) covered in hits of different bacterial species was recorded for each of the genes, as well as the highest percentage identity (\%ID), provided one of these percentages exceeded $50 \%$.

In Vitro Ames Test with a Cell-Free Spent Supernatant of EcN. Escherichia coli strain Nissle 1917 (EcN) and its supernatant were obtained from the producer, Ardeypharm GmbH, Herdecke, Germany. Classical Ames test was performed according to the international guidelines [17] at Eurofins BioPharma Product Testing, Munich. The test item was a cell-free spent supernatant obtained from $\mathrm{EcN}$, batch \# 729210 containing $1-2 \cdot 10^{11}$ colony forming units (CFU)/ $\mathrm{mL}$. It was used in 5 concentrations: undiluted and diluted $1: 2,1: 10,1: 20$, and 1:100 in sterile salt solution (SS, $40 \mathrm{mM}$ $\mathrm{NaCl}, 30 \mathrm{mM} \mathrm{KCl}, 0.8 \mathrm{mM} \mathrm{MgSO}_{4}, 1.4 \mathrm{mM} \mathrm{CaCl}_{2}, 0.1 \mathrm{mM}$ $\mathrm{MgCl}_{2}$, and $\mathrm{pH}$ 7.0). This corresponded to a volume of cellfree supernatant of $100 \mu \mathrm{L}, 50 \mu \mathrm{L}, 10 \mu \mathrm{L}, 5 \mu \mathrm{L}$, and $1 \mu \mathrm{L}$ tested per agar plate. The highest tested dose would exceed the recommended daily maximum intake of the $\mathrm{EcN}$ product administered to young children by 20 -fold.

The test was performed according to the published guidelines [17, 23-25] with Ames reporter bacteria of Salmonella enterica serovar Typhimurium strains TA98, TA100, TA1535, TA1537, and TA102. The test was performed without and with metabolic activation. For the latter, S9 mix (Trinova Biochem $\mathrm{GmbH}$, Gießen, Germany) was prepared according to the literature $[11,17]$. A solvent control of SS and a negative control (sterile water) were included. As a positive control for Ames reporter strains TA100 and TA1535 without metabolic 
activation sodium azide (Sigma) was included at a concentration of $10 \mu \mathrm{g} /$ plate. The positive control 4-nitro-o-phenylenediamine (Fluka) dissolved in dimethyl sulfoxide (DMSO) was used at $10 \mu \mathrm{g} / \mathrm{plate}$ for reporter strain TA98 and at $40 \mu \mathrm{g} / \mathrm{plate}$ for reporter strain TA1537. The positive control for reporter strain TA102 without metabolic activation was $1 \mu \mathrm{L} /$ plate methyl methanesulfonate. The positive control for all Ames reporter strains in combination with $\mathrm{S} 9$ metabolic activation was 2-aminoanthracene (Aldrich) dissolved in DMSO. This was used at a concentration of $2.5 \mu \mathrm{g} /$ plate, except for strain TA102 that required $10 \mu \mathrm{g} /$ plate.

Following standard procedures, $100 \mu \mathrm{L}$ of the test item or control was mixed with $500 \mu \mathrm{L} \mathrm{S} 9$ mix for tests with metabolic activation or with $500 \mu \mathrm{L}$ S9 mix substitution buffer for tests without metabolic activation, $100 \mu \mathrm{L}$ Ames reporter strain (approx. $10^{9} \mathrm{CFU} / \mathrm{mL}$, early stationary phase), and $2 \mathrm{~mL}$ of molten top agar $(0.7 \%$ agar, $0.6 \% \mathrm{NaCl}, 0.05 \mathrm{mM}$ histidine/biotin at $44-48{ }^{\circ} \mathrm{C}$ ). This mixture was poured on Vogel Bonner glucose minimal agar plates. For experiments with pre-incubation, the mixture of test material, Ames reporter strain, and S9 mix or substitution buffer was pre-incubated for $60 \mathrm{~min}$ at $37^{\circ} \mathrm{C}$ prior to adding the molten top agar. All tests were performed in triplicate. After $48 \mathrm{~h}$ of incubation at $37{ }^{\circ} \mathrm{C}$, revertant colonies were counted using a ProtoCOL counter (Meintrup DWS Laborgeräte, Herzlake, Germany); manual counting was used for strains TA1535 and TA1537 that have a low spontaneous mutation frequency. The number of induced revertants was compared to the number of spontaneous revertant colonies on solvent control plates and to the number of revertants induced by the mutagenic reference substance on positive control plates. A substance is considered as mutagenic if it results in reproducible increase in the number of revertant colonies in one or more reporter strains, and if the number of revertants is at least double the number of spontaneous revertant colonies.

In Vitro Modified Ames Test with Viable EcN. Live EcN bacteria from overnight cultures $\left(16-18 \mathrm{~h}, 37^{\circ} \mathrm{C}\right.$ in nutrient broth) were centrifuged ( $8 \mathrm{~min}, 5000 \mathrm{rpm})$, and cell pellets were washed twice with phosphate buffer and resuspended in sterile water to $1-2 \cdot 10^{9} \mathrm{CFU} / \mathrm{mL}$. Recovered supernatant of the $\mathrm{EcN}$ overnight culture was sterile filtrated to obtain a cell-free supernatant containing metabolic products of $\mathrm{EcN}$. These test items were tested in a modification of the classical Ames test. The modification included addition of 20 or $24 \mu \mathrm{g} / \mathrm{mL}$ streptomycin (Roth, Karlsruhe, Germany) to the Vogel Bonner glucose minimal agar plates and to the top agar, to inhibit the growth of EcN but not inhibiting the Ames reporter strains (this modification was omitted for testing spent supernatant). The tests were performed with Ames test strains TA98, TA1537, and TA100 as reporter strains. Pilot experiments confirmed this selective inhibition of EcN, whereby the presence of the antibiotic resulted in a background lawn similar to streptomycin-free plates and normal numbers of spontaneous revertants of Ames reporter strains, with the exception of reporter strain TA100, which showed a small reduction of spontaneous revertant colonies (data not shown). All tests were performed with preincubation in the absence of metabolic activation as described above, with some modifications: pre-incubation was performed for $20 \mathrm{~min}$ only, and as a positive control, 4nitroquinoline-1-oxid (Sigma, final concentration $0.5 \mu \mathrm{g}$ / plate) was used for TA98 and TA100, and 9-Aminoacridin (Merck, $60 \mu \mathrm{g} /$ plate) for TA1537. Sterile distilled water served as the negative control. Each sample was plated twice on minimal agar plates and all experiments were performed in triplicate with mean results plus standard deviation reported here.
Test Item for the In Vivo Mammalian Alkaline Comet Assay. The in vivo Mammalian Alkaline Comet Assay was performed with EcN viable bacteria containing an average of $1.1 \cdot 10^{11} \mathrm{CFU} / \mathrm{mL}$ (range: $7.5 \cdot 10^{10}-1.6 \cdot 10^{11}$ ). Three dose levels of $\mathrm{EcN}$ were applied: $10^{11} \mathrm{CFU} / \mathrm{mL}$ (undiluted $\mathrm{EcN}$ ), $10^{10} \mathrm{CFU} / \mathrm{mL}$ (1:10 dilution in SS), and $10^{9} \mathrm{CFU} / \mathrm{mL}(1: 100$ dilution). Sterile SS served as vehicle control. In all rat experiments, single doses of $1 \mathrm{~mL}$ bacterial suspension or vehicle control were given per animal on consecutive days.

Animal Experiments. The animal experiments were conducted at BSL Munich, Germany, in an AAALACaccredited laboratory.

Young healthy SPF Wistar rats $(\mathrm{Crl}$ : WI(Han)) of either sexes were obtained at 6-8 weeks of age from a professional breeder (Charles River, Sulzfeld, Germany) and allowed to acclimatize for at least 5 days in groups of 1 to 3 randomly selected animals per cage, sorted for sex. At the age of 7-9 weeks, EcN was orally administered to the animals via gavage. A negative control group (NC) consisted of 5 untreated animals (only in Experiment A), and a vehicle control group (VC) included 5 animals dosed with SS. A positive control group (PC) consisted of 5 animals treated with ethyl methanesulfonate (Sigma-Aldrich/Merck, Darmstadt, Germany) in $0.9 \% \mathrm{NaCl}$, at a single dose of $100 \mathrm{mg}$ per kg body weight (bw) in Experiment A and $300 \mathrm{mg} / \mathrm{kg}$ bw in Experiments B and C. All animals had free access to water and feed during the course of the experiment.

For the pilot dose-range finding experiment, various doses of EcN suspension were tested to identify the maximum tolerated dose (MTD), defined as the dose that induces the first slight toxic effects with respect to the duration of the main study period. Three animals of each sex were used for this, and since no differences could be determined between male and female animals, all other experiments were performed with male rats only.

Since no toxic effect was detected after application of a maximum feasible dose of $1 \mathrm{~mL}$ per day with $1 \cdot 10^{11} \mathrm{CFU} /$ $\mathrm{mL}$, this dose was chosen as MTD. A higher dose would not be feasible as the bacterial suspension would become too viscous to be applied in the manner required.

The marketed EcN product used in infants and children up to the age of 12 (Mutaflor ${ }^{\circledR}$ Suspension) is applied at a daily dose of up to $5 \mathrm{~mL}$ containing $10^{8} \mathrm{CFU} / \mathrm{mL}$. This corresponds to a daily dose of $1.1 \cdot 10^{7} \mathrm{CFU} / \mathrm{kg}$ bw for a child of $45 \mathrm{~kg}$, or 10 times that much for an infant of $4.5 \mathrm{~kg}$. The latter refers to $1.67 \cdot 10^{7} \mathrm{CFU}$ for a rat weighing $0.15 \mathrm{~kg}$ on average. We exceeded this dose with a factor of 6000 .

For determination of acute oral toxicity $[24,26]$ short-term exposure experiments were performed with 6 groups (Experiment A) and 3 groups (Experiment B) of 5 rats; dosage and treatment are summarized in Table 1. All animals except for groups 2 and 8 (positive controls) were treated orally by gavage on days 1 and 2 and were sacrificed $4 \mathrm{~h}$ after the second dose on day 2 . The positive control groups only received a single dose on day 2. For long-term exposure (Experiment C), the daily dose was provided on 28 consecutive days according to the guidelines [27] (Table 1). During the period of administration, the animals were carefully observed for signs of toxicity by qualified staff. Following euthanasia at the end of the experiment, the relevant tissues were sampled.

To verify that $\mathrm{EcN}$ had reached the target organs and to prove a continuous exposure, animal feces were sampled group-wise at various time points during all experiments and analyzed for the presence of EcN using cultural methods followed by a strain-specific PCR as previously described [28].

Clinical Scoring. All animals were observed for clinical signs during the experiment. General clinical observations were performed at least once a day at approximately the same 
Table 1. Experiments for in vivo assessment, with 2-day and 28-day colonization

\begin{tabular}{|c|c|c|c|}
\hline Exp. & Group no. $n=5$ animals & Treatment & Dose (in $1 \mathrm{~mL}$ ) per animal, timing \\
\hline \multirow{6}{*}{ Exp. A } & 1 & Negative control & untreated \\
\hline & 2 & Positive control & $100 \mathrm{mg}$ EMS/kg bw on day 2 \\
\hline & 3 & Vehicle control & Sterile salt solution on days 1 and 2 \\
\hline & 5 & $\mathrm{EcN}$ & 0.10 MTD: $10^{10} \mathrm{CFU} \mathrm{EcN}$ on days 1 and 2 \\
\hline & 6 & $\mathrm{EcN}$ & MTD: $10^{11}$ CFU EcN on days 1 and 2 \\
\hline & 7 & Vehicle control & Sterile salt solution on days 1 and 2 \\
\hline \multirow[t]{4}{*}{ Exp. B } & 8 & Positive control & $300 \mathrm{mg}$ EMS $/ \mathrm{kg}$ bw (in $10 \mathrm{~mL} / \mathrm{kg}$ ), on day 2 \\
\hline & 9 & $\mathrm{EcN}$ & MTD: $10^{11}$ CFU EcN on days 1 and 2 \\
\hline & 10 & Vehicle control & Sterile salt solution daily on days $1-28$ \\
\hline & 11 & Positive control & $300 \mathrm{mg} \mathrm{EMS} / \mathrm{kg}$ bw (in $10 \mathrm{~mL} / \mathrm{kg}$ ) on day 28 \\
\hline \multirow[t]{3}{*}{ Exp. C } & 12 & $\mathrm{EcN}$ & 0.01 MTD: $10^{9}$ CFU EcN daily on days $1-28$ \\
\hline & 13 & $\mathrm{EcN}$ & 0.10 MTD: $10^{10} \mathrm{CFU}$ EcN daily on days $1-28$ \\
\hline & 14 & $\mathrm{EcN}$ & MTD: $10^{11}$ CFU EcN daily on days $1-28$ \\
\hline
\end{tabular}

Exp: Experiment. EcN: E. coli Nissle 1917. MTD: maximum tolerated dose. EMS: ethyl methanesulfonate.

time in consideration of the peak period of anticipated effects after dosing. The health condition of the animals was recorded and twice, daily all animals were observed for morbidity and mortality.

Body weight was recorded from all animals before assignment to the experimental groups, on the first day of administration and weekly during the long-term application. All animals subjected to necropsy were additionally weighed on the day of necropsy.

Intestinal Cell Preparation. Following euthanasia, the selected tissues were removed and kept in ice-cold mincing buffer (Hank's Balanced Salt Solution containing $20 \mathrm{mM}$ EDTA, pH 7.5, with 10\% DMSO added before use). The tissues were rinsed with this buffer to remove residual blood and kept on ice until further processing. A portion of the small intestine and the upper and lower parts of the colon were minced with a pair of scissors and the cell suspension was filtered through a mesh $(70 \mu \mathrm{m})$ to yield a single cell suspension. The cell number of isolated cells was adjusted by determination using the trypan blue dye exclusion, test and approximately $2-4 \times 10^{5}$ cells were pelleted by centrifugation. These cell pellets were used to prepare comet slides.

Comet Assay. The Comet assay was performed according to the guidelines [18]. The slides were pre-coated with $0.8 \%$ normal-melting agarose in a phosphate buffer $(137 \mathrm{mM} \mathrm{NaCl}$, $2.7 \mathrm{mM} \mathrm{KCl}, 1.5 \mathrm{mM} \mathrm{KH} \mathrm{PO}_{4}, 8.1 \mathrm{mM} \mathrm{Na}_{2} \mathrm{HPO}_{4}, \mathrm{pH}$ 7.4). The cell pellets were resuspended in $225-\mu \mathrm{L}$ warm $\left(40{ }^{\circ} \mathrm{C}\right)$ $0.8 \%$ low-melting agarose in a phosphate buffer. Approximately $8-12.5 \cdot 10^{4}$ cells were embedded in lowmelting agarose on the slides in one layer, covered with a cover slip and cooled for $30 \mathrm{~min}$ on ice.

The slides were incubated in pre-chilled lysing solution (2.5 M NaCl, $100 \mathrm{mM}$ EDTA, $10 \mathrm{mM}$ TrisHCl, $1 \%$ sodium lauroyl sarcosine, with freshly added $10 \%$ DMSO and $1 \%$ Triton-X100) at $2-8{ }^{\circ} \mathrm{C}$ overnight for lysis of the cells and the release of DNA, rinsed in pre-chilled neutralization solution (400 mM TrisHCl, $\mathrm{pH} 7.5$ ), and then incubated for $20 \mathrm{~min}$ in alkaline solution ( $300 \mathrm{mM} \mathrm{NaOH}, 1 \mathrm{mM}$ EDTA, $\mathrm{pH}>13)$ to unwind the DNA. Electrophoresis of the slides was performed in the same alkaline solution for $30 \mathrm{~min}$. Following neutralization by washing three times with a neutralization buffer for $5 \mathrm{~min}$, the slides were treated for $20 \mathrm{~min}$ in ice-cold ethanol and air-dried before DNA was stained with $70 \mu \mathrm{L}$ ethidium bromide $(20-40 \mu \mathrm{g} / \mathrm{mL})$. A fluorescence microscope with $200 \times$ magnification coupled with a camera was used to analyze the comets, and Comet Software (Perceptive Instrument System IV, Instem, Staffordshire, UK) was used for blinded evaluation.

Scores were classified into 3 categories: scorable, non-scorable, and "hedgehog" (images consisting of a small or non- existent head and large diffuse tails as the result of heavily damaged cells). To avoid artefacts, only scorable cells of at least 150 cells per sample were scored. The tail intensity was expressed as a percentage of the total intensity in the nucleus.

The median \% tail DNA for each slide was determined, and the mean of the median value was calculated for each animal. These individual mean values were used to calculate a group mean. Statistical analysis was done by one-way ANOVA with Dunnett's test and by unpaired $t$-test with Welch's correction, and in both cases, normality test by Kolmogorov-Smirnov was used.

Ethics. In accordance to German animal protection laws, the study type was reviewed and accepted by local authorities, subjected to the Ethical Review Process and authorized by the Bavarian Animal Welfare Administration.

\section{Results}

Conservation of Key Pks Genes in Bacterial Species. To assess the conservation of $p k s$ genes among bacterial species, BlastP searches were performed with amino acid sequences extracted from the genome of EcN. Although the pks locus is not present in every sequenced member of a bacterial species, when present, it is usually strongly conserved within that species (results not shown). The degree of conservation of key genes between bacterial species is reported by 2 parameters in Figure 1: top hits obtained with BlastP are shown with the percentage of the query length producing overlap $(\% \mathrm{QL}$, the query being the EcN gene) and by the percentage of identity within the found overlap (\%ID). As can be seen, strong conservation was found for members of Enterobacter hormaechei (a colonizer of the human gut [29], Klebsiella pneumoniae and Klebsiella aerogenes, and Citrobacter koseri, an opportunistic pathogen. Less strongly conserved genes were detected in Klebsiella oxytoca, Erwinia oleae, and Serratia marcescens, while the genes of Frischella perrara (a gut symbiont of honey bees) are the least conserved (Figure 1).

In Vitro Testing of EcN Spent Supernatant by the Ames Test. The results of the classical Ames test, performed with 5 different Ames reporter strains, are summarized in Figure 2. The test item was tested at 5 concentrations, the highest of which (undiluted supernatant) represents a 20 -fold excess of the dose under recommended use for infants. The tests were performed in the absence and presence of metabolic activation, while all tests were carried out with and without pre-incubation of the test material with the reporter strain. All tests performed with 5 concentrations of the cell-free spent supernatant were negative, with non-significant differences to the negative and solvent controls in all cases. All positive 

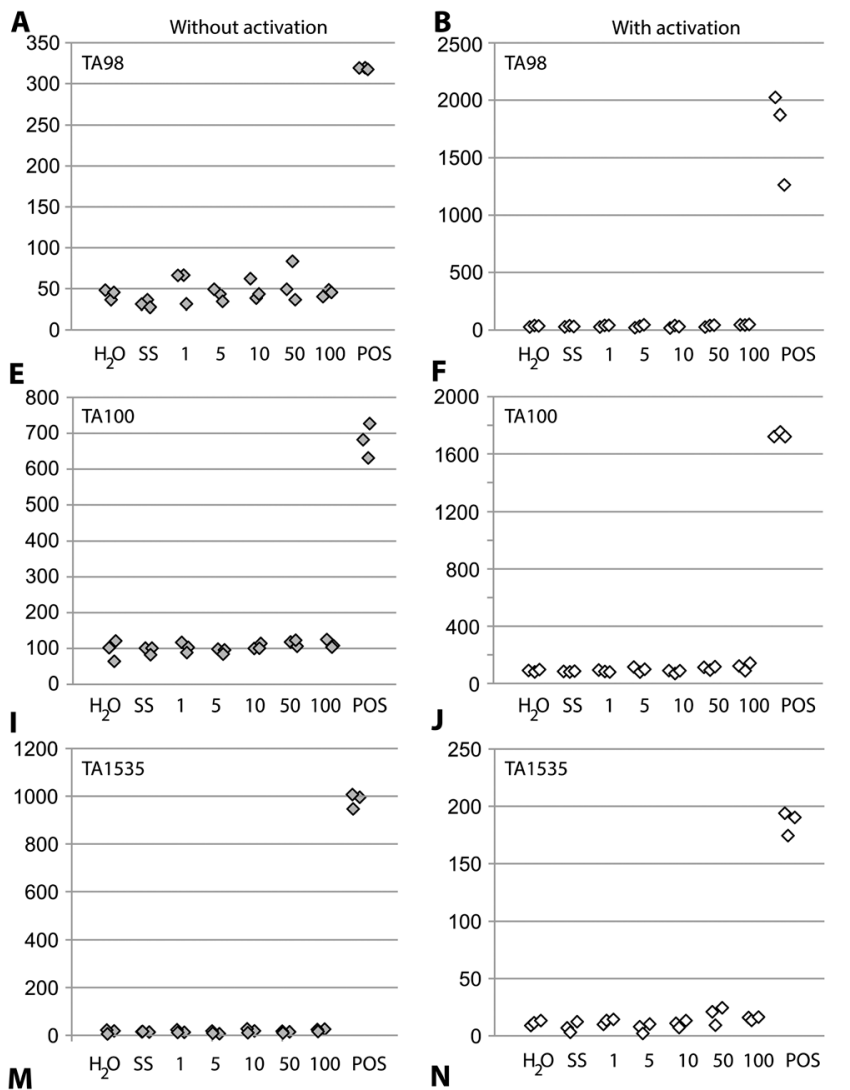

$\mathbf{M}$
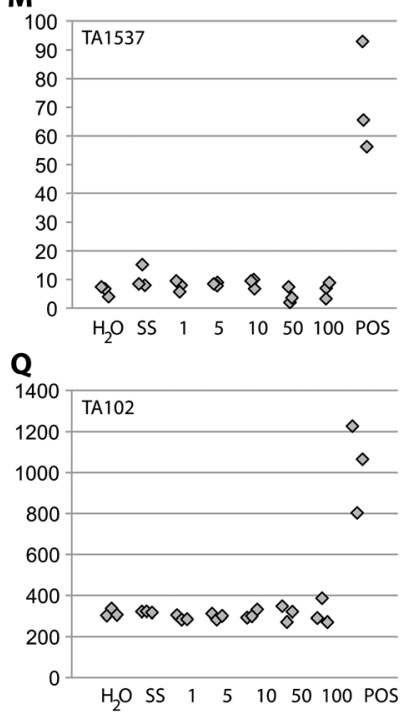
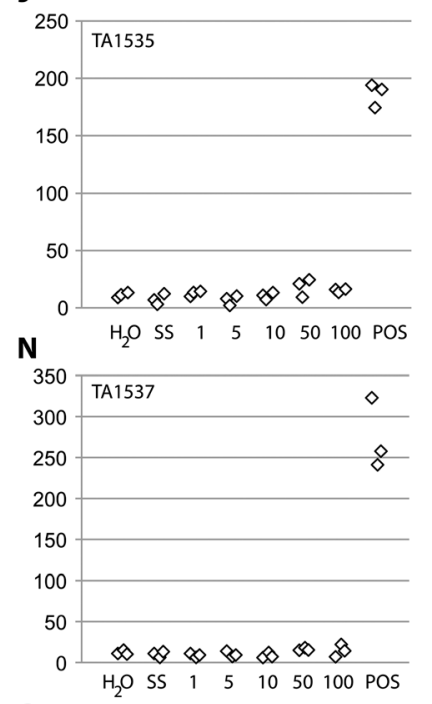

\section{$\mathbf{R}$}

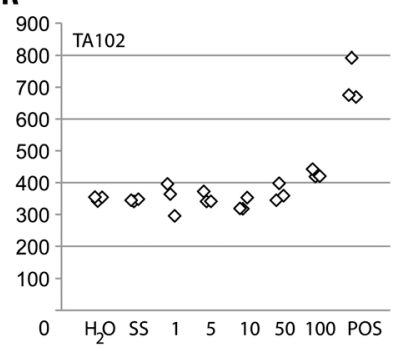

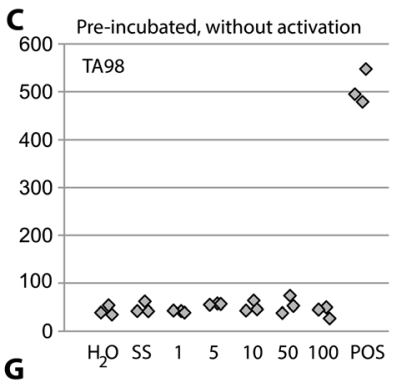
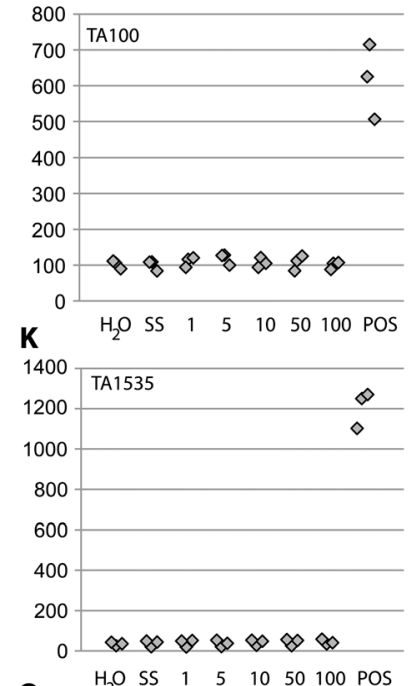

O

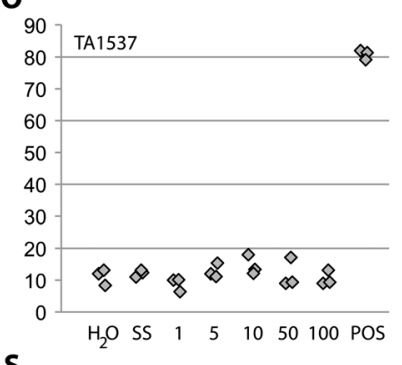

\section{$\mathbf{S}$}

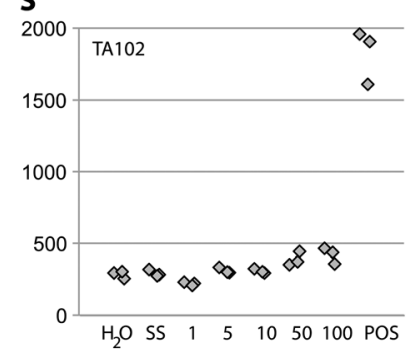

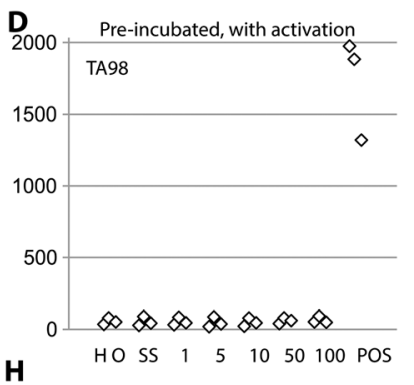

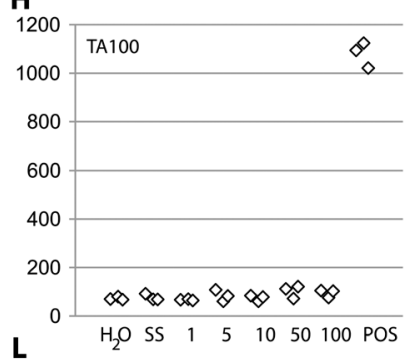

$1 6 0 \longdiv { \text { TA1535 } }$

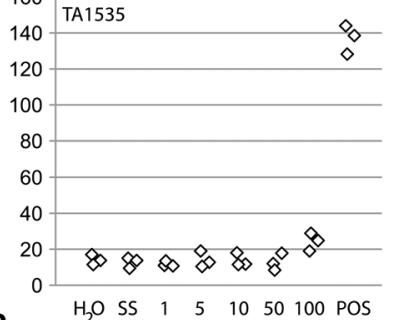

P 90

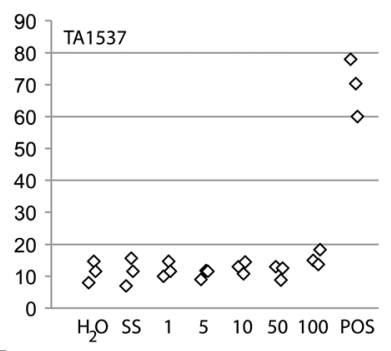

$\mathbf{T}$

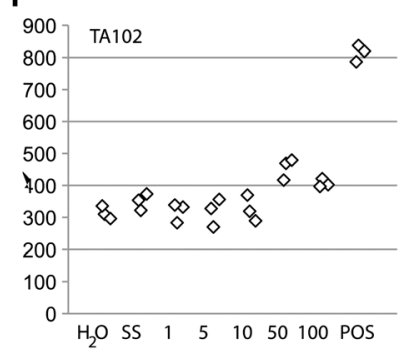

Figure 2. In vitro assessment of mutagenic activity of spent $\mathrm{EcN}$ supernatant by the classical Ames Test. Five concentrations (as indicated, in $\mu \mathrm{L}$ supernatant per plate) were tested, and the resulting numbers of revertant colonies per plate are shown for 5 Ames reporter strains: TA98 (A-D), TA100 (E-H), TA1535 (I-L), TA1537 (M-P), and TA102 (Q-T). The 2 left columns show experiments without pre-incubation (without and with metabolic activation), and the 2 right columns represent data with pre-incubation. Grey symbols are used for experiments without metabolic activation and, white symbols represent results obtained with S9-mix activation. $\mathrm{H}_{2} \mathrm{O}$ : negative control, SS: sterile salt solution, vehicle control. POS: positive control as specified in the Materials and Methods section

controls resulted in highly significant readings $(P<0.001)$. Thus, no mutagenic or cytotoxic activity could be demonstrated for the spent supernatant of $\mathrm{EcN}$ assessed by the classical Ames test, under all tested conditions.

In Vitro Testing of Viable EcN by the Modified Ames Test. Since colibactin-induced toxicity depends on direct cell contact [3], we also tested mutagenic activity of viable $\mathrm{EcN}$ bacteria by pre-incubation with Ames reporter strains, in a modified Ames tests (see Materials and Methods). By addition of streptomycin to the agar plates, the growth of $\mathrm{EcN}$ could be suppressed without affecting the Ames reporter strains. The results are summarized in Figure 3. Only the positive controls resulted in a significant increase of revertants. All other test items produced revertant colonies in numbers not significantly different from those obtained with the solvent control. Thus, even while direct cell contact was enabled, EcN failed to exert any mutagenic effect on the Ames reporter strains.

In Vivo Genotoxicity of EcN in Rats as Determined by the Comet Assay. In vivo genotoxic effects of EcN were tested in rats by oral application for 2 days or 28 days, respectively, followed by the Comet assay to analyze the exposed intestinal epithelial cells. Microbiological and PCR analysis of the rats' feces evidenced that those animals that had received the test item carried living $\mathrm{EcN}$ in their feces throughout the experiments (results not shown). This indicates that, as expected, live $\mathrm{EcN}$ reached its site of action, the gut, and 
A

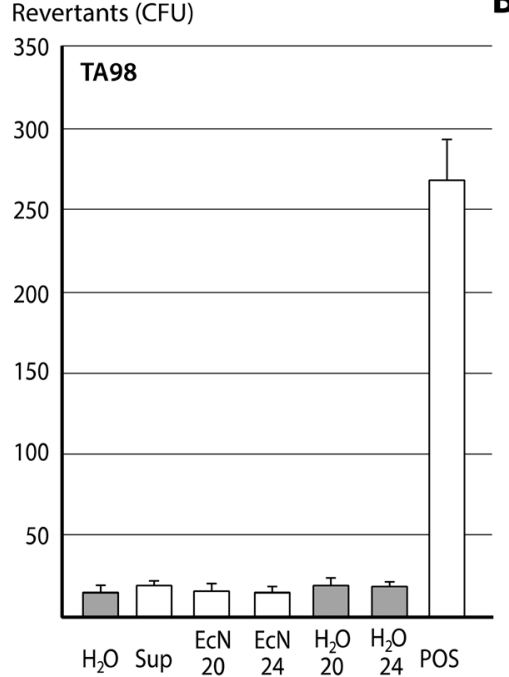

B

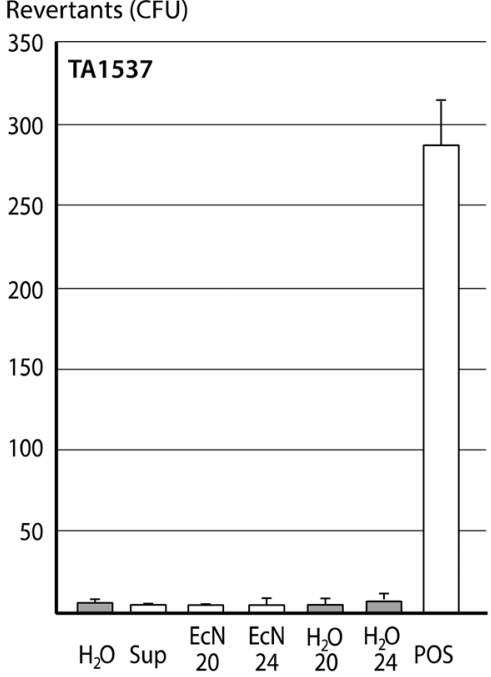

C Revertants (CFU)

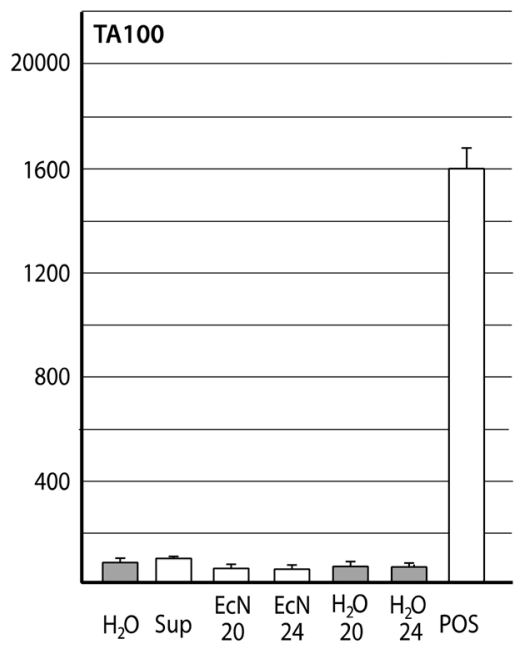

Figure 3. In vitro mutagenic activity assessment by the modified Ames test of live EcN cells and its spent supernatant. Shown are the numbers of revertant colonies obtained per plate for Ames tester strain TA98 (A), strain TA1537 (B), and strain TA100 (C). To inhibit growth of EcN, 2 concentrations $(20$ and $24 \mu \mathrm{g} / \mathrm{L})$ of streptomycin were added to the minimal medium as indicated. Sup: spent supernatant of EcN culture. EcN: live $E$. coli Nissle 1917 with $1-2 \times 10^{8}$ CFU per plate. $\mathrm{H}_{2} \mathrm{O}$ : sterile water served as solvent control for spontaneous revertants. POS: positive control as specified in the Materials and Methods section

verified that the target tissues had indeed been exposed to the test item.

During application, no clinical signs of toxicity were observed with the test animals, and the post-mortem histopathological examination showed no significant findings (results not shown).

Three sites of the gastrointestinal tract were assessed with the Comet assay: the small intestine, and the upper and lower parts of the colon. The results are presented in Table 2. The positive controls resulted in statistically significant $(p<0.05)$ DNA damage of cells from all 3 tested tissues, in all 3 experiments (Table 2). For the highest tested dose of $10^{11}$ CFU, a significant $(p=0.0032)$ decrease in scores for DNA damage was observed in cells collected from the small intestine, in Experiment B only (Table 2). The comet tail intensity for all other tissues from the $\mathrm{EcN}$ application groups was in the same range as the negative (vehicle) control. In none of the tissues from the EcN application group, the comet tail intensity was increased, which would have been an indication of induced DNA strand breaks. The distribution of the findings per animal group is shown as Box-and-Whisker plots in Figure 4. The results clearly show that EcN did not induce DNA damage in the intestinal tissue of the exposed animals.

\section{Discussion}

A number of in vitro and in vivo tests are available to test the possible mutagenicity or genotoxicity of a given compound. All these tests have advantages and disadvantages, in part depending on the mechanism by which a potential mutagen might cause DNA damage, as discussed elsewhere [30]. The Ames test makes use of certain reporter Ames strains to detect mutagenicity by means of bacterial reverse mutation, which excludes its application to test mutagenic properties of live bacteria. The test is applicable to cell-free supernatant, but in the case of colibactin, testing spent supernatant may be less relevant. It has been described that colibactin, when produced by $E$. coli, is rapidly inactivated upon secretion and that direct cell contact is needed for genotoxic effects [3]. Thus, the finding that $\mathrm{EcN}$ spent supernatant did not have any detectable mutagenic properties was not fully informative.

We were able to overcome the practical limitation of the classical Ames test by the addition of a selective antibiotic, which allowed the testing of viable EcN without affecting the spontaneous reversion rates of the Ames reporter strains too much. Again, no mutagenic activity could be demonstrated for $\mathrm{EcN}$, although now EcN was co-incubated with the Ames reporter strains, and direct cell contact was enabled throughout the experiment. These findings demonstrate that neither living $\mathrm{EcN}$ bacteria nor its metabolites show genotoxic, cytotoxic, or mutagenic activity in the Ames test.

It has been suggested that colibactin produces DNA strand breaks, which are excellently detectable with the Comet assay. Compared to other in vivo tests, the Comet assay is superior, as it could identify $92 \%$ of probable or confirmed human carcinogens, a result that was better than any of the other tests

Table 2. Comet Assay, mean tail DNA intensities [\%] in cells from the small intestine, upper and lower part of the colon of exposed rats from the three experiments

\begin{tabular}{|c|c|c|c|c|c|c|c|c|c|}
\hline & \multicolumn{3}{|c|}{$\begin{array}{c}\text { Small intestine } \\
\text { (group means } \pm \text { standard deviation) }\end{array}$} & \multicolumn{3}{|c|}{$\begin{array}{c}\text { Upper colon } \\
\text { (group means } \pm \text { standard deviation) }\end{array}$} & \multicolumn{3}{|c|}{$\begin{array}{c}\text { Lower colon } \\
\text { (group means } \pm \text { standard deviation) }\end{array}$} \\
\hline & Exp. A & Exp. B & Exp. C & Exp. A & Exp. B & Exp. C & Exp. A & Exp. B & Exp. C \\
\hline Negative control & $1.87 \pm 0.34$ & n.d. & n.d. & $3.19 \pm 0.74$ & n.d. & n.d. & $2.47 \pm 1.15$ & n.d. & n.d. \\
\hline Positive control & $5.99 \pm 2.37^{\mathrm{a}}$ & $17.83 \pm 7.11^{\mathrm{a}}$ & $14.94 \pm 7.17^{\mathrm{a}}$ & $7.15 \pm 0.90^{\mathrm{a}}$ & $12.72 \pm 5.92^{\mathrm{a}}$ & $14.07 \pm 5.97^{\mathrm{a}}$ & $6.42 \pm 1.65^{\mathrm{a}}$ & $12.99 \pm 3.73^{\mathrm{a}}$ & $14.19 \pm 8.95^{\mathrm{a}}$ \\
\hline Vehicle control & $2.82 \pm 0.72$ & $3.77 \pm 0.85$ & $3.81 \pm 0.67$ & $3.25 \pm 1.14$ & $1.87 \pm 0.63$ & $2.88 \pm 1.46$ & $2.84 \pm 1.47$ & $1.10 \pm 0.65$ & $2.57 \pm 1.53$ \\
\hline $0.01 \mathrm{MTD}$ & $1.28 \pm 0.20$ & n.d. & $2.54 \pm 0.97$ & $3.70 \pm 0.78$ & n.d. & $2.69 \pm 1.70$ & $2.17 \pm 1.02$ & n.d. & $3.27 \pm 1.21$ \\
\hline $0.1 \mathrm{MTD}$ & $1.24 \pm 0.66$ & n.d. & $2.95 \pm 2.09$ & $3.55 \pm 1.29$ & n.d. & $3.15 \pm 1.84$ & $1.13 \pm 0.59$ & n.d. & $2.95 \pm 0.99$ \\
\hline 1 MTD & $0.98 \pm 0.48$ & $1.75 \pm 0.60^{\mathrm{b}}$ & $3.94 \pm 2.89$ & $3.35 \pm 0.82$ & $1.59 \pm 0.75$ & $3.48 \pm 2.36$ & $2.28 \pm 1.59$ & $0.82 \pm 0.37$ & $3.90 \pm 2.59$ \\
\hline
\end{tabular}

${ }^{\text {a }}$ Significantly increased compared to vehicle control.

${ }^{\mathrm{b}}$ Significantly decreased compared to vehicle control. 
that were compared [31]. Moreover, rats are more easily colonized by $E$. coli than mice, so we consider this animal model to be the most suitable test for the detection of genotoxic activity. Even though the highest tested dose exceeded the daily recommended dose of EcN to infants by a factor of 6000, no cytotoxic or mutagenic effects could be demonstrated, whether the animals were exposed for 2 days or for 28 days.

The findings reported here are in strong contrast with the literature, in which the mutagenicity of colibactin, encoded by the pks locus, is hardly doubted. This presumed mutagenicity was demonstrated in a number of in vitro and in vivo models [3-9]. One in vitro model was used to demonstrate that outer membrane vesicles (OMV) from EcN could increase the number of DNA strand breaks in cultured HT-29 cells as demonstrated by the Comet assay, and although those same vesicles did not display cytotoxic activity towards the cells, their proliferation was slightly reduced [32]. The authors speculate that the formed OMVs can pass the intestinal mucus barrier in humans and are taken up by epithelial cells, which would contribute to the beneficial activity of EcN. If, in our experiments, vesicles were formed in the intestine of the rats, they did not generate double-strand DNA breaks, as our data revealed no evidence of DNA damage at all. In line with this observation is the report that a mucus layer attenuates any genotoxic effect of colibactin, as was demonstrated by in vitro experiments [33].

Most in vivo experiments that demonstrated genotoxic effects of colibactin were performed with pks-positive E. coli strains other than EcN. For instance, a mouse model with germ-free IL-10 $0^{-/-}$knockout mice was used to induce colitis by mono-colonization with $E$. coli strain NC101, after which treatment with the carcinogen azoxymethane resulted in tumors. A carcinogenic effect that depended on the presence of the pks locus was shown for the used E. coli strain [5, 34]. In this model, even E. coli strains lacking the pks locus caused cancer, but those tumors appeared later and were both fewer and smaller than was observed in animals monocolonized by pks-positive E. coli.
Experiment A, 2 days
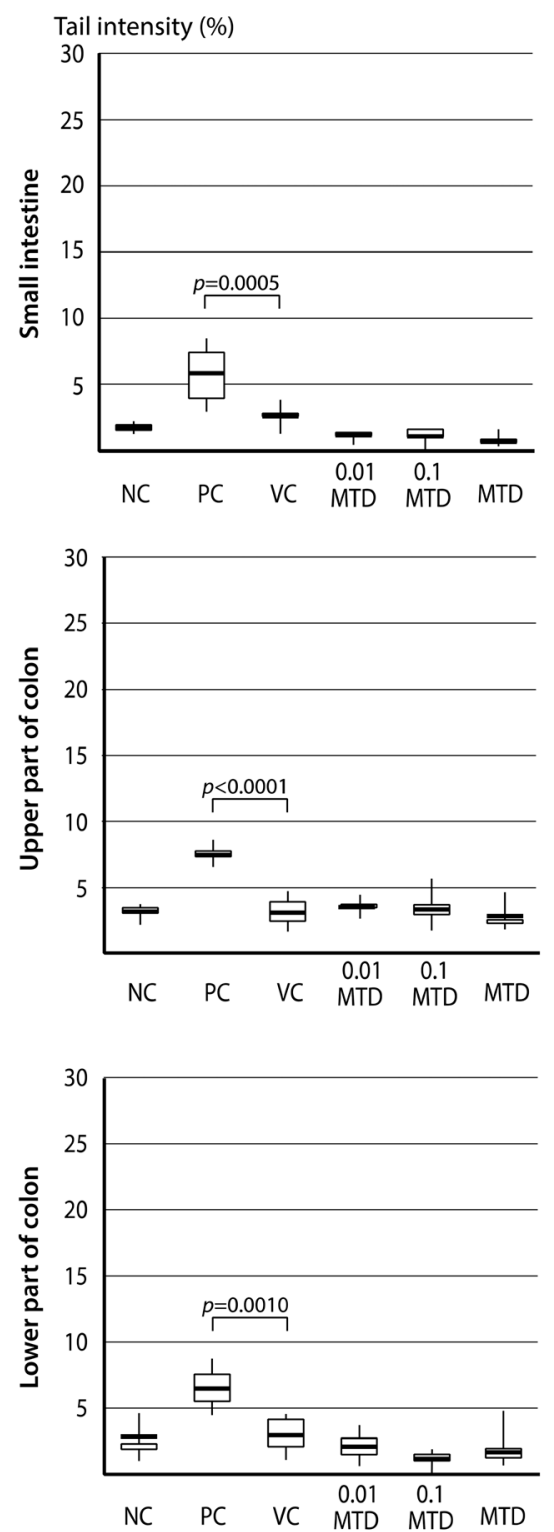

Experiment B, 2 days
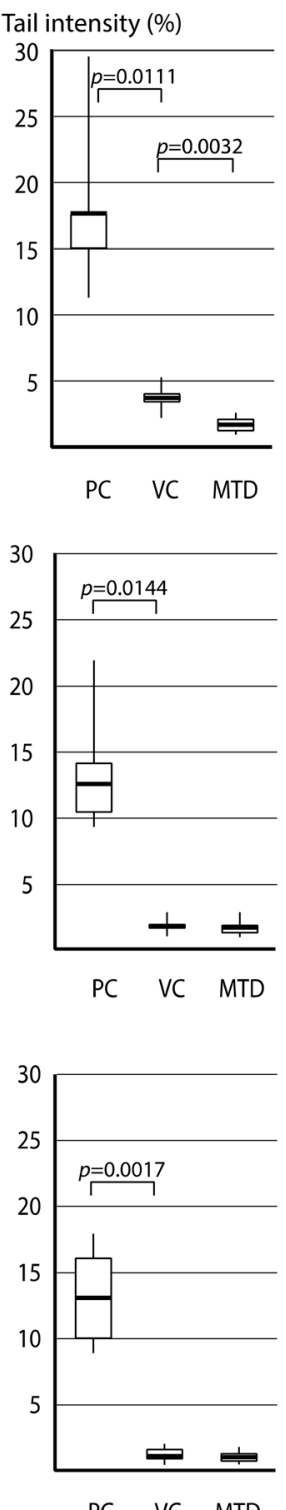

PC VC MTD
Experiment $C, 28$ days
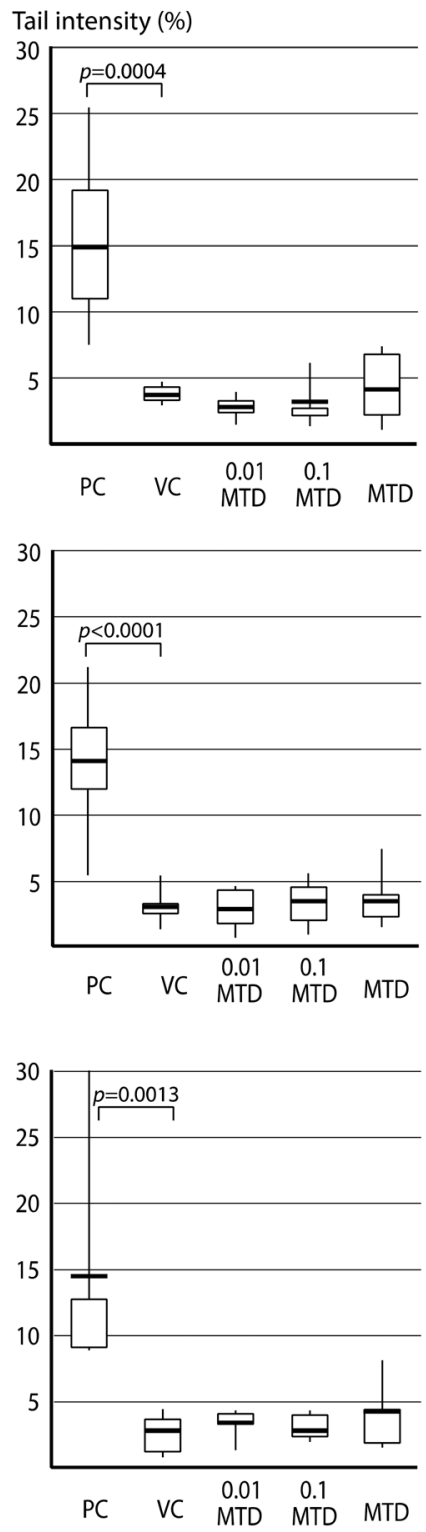

Figure 4. In vivo assessment of mutagenic activity of EcN, shown as tail intensity in the Comet Assay. Rats received EcN for 2 days (Experiments A, left column, and B, middle column) or for 28 days (Experiment C, right column), and DNA damage was assessed by the Comet assay with cells from the small intestine (top), upper part of the colon (middle), and lower part of the colon (bottom). Statistical significant findings compared to the vehicle control are indicated with corresponding $p$-values. PC: positive control. VC: vehicle control. NC: untreated animals. MTD: maximum tolerated dose 
Another model compared $\mathrm{C} 57 \mathrm{~B} / 6 \mathrm{~J}_{-} \mathrm{Apc}^{-/+}$mice (Min mice) with wild-type animals that were treated with streptomycin prior to inoculation. These mice were colonized for 50 days with either a $p k s$-positive or a K12 (pks-negative) E. coli strain, or they were not infected; $50 \%$ of the non-infected control animals developed colonic polyps, compared to $57 \%$ of K12-colonized and $92 \%$ of the animals colonized with the pks-positive strain. Of note, the wild-type animals did not develop neoplasia, despite being colonized with high levels of pks-positive E. coli [7].

A third murine model, that utilized colon loops, was used to demonstrate a rapid DNA-damaging effect of pks-bearing E. coli strain SP15 [4]. It should be noted that all these assays are rather artificial, and none are standard procedures to determine carcinogenic activity of an agent. The gold standard for such testing is the in vivo Comet Assay, with exposed tissues being investigated in a mammalian test system. Even for strains that stably produce colibactin, their genotoxicity is not easily demonstrated. A recent publication was able to demonstrate colibactin activity by means of the Comet Assay after exposing HeLa cells to a pks-positive $E$. coli strain, but only when the cells were additionally treated by 8 Gy gamma-irradiation. Interestingly, the comets produced in the presence of the colibactin-positive $E$. coli were significantly shorter than those obtained with cells irradiated in the absence of the bacteria. The authors interpreted this to be due to DNA cross linking [35].

Not all literature agrees with the in vivo effect of colibactin. It was demonstrated that at high multiplicity of infection, $E$. coli strains expressing $p k s$ can actually suppress the proliferation of tumor cells in a murine model using xenografts of $E$. coli-infected HC116 cells [36]. Since EcN bacteria are attracted to tumor tissue, this ability can even be employed for tumortargeting bacterial therapy [37]. The authors of that publication demonstrated that genetically modified EcN overproducing colibactin suppressed tumors more strongly in a mouse model [37]. In our experiments, we detected significantly less DNA damage in one group of animals infected for 2 days with the maximum tolerated dose of EcN (Experiment B), but this finding was not replicated in the other experiments, so we are reluctant to conclude that the effect is real. Our data do, however, reproducibly report the lack of mutagenic activity of $\mathrm{EcN}$ in rats, as the bacteria did not induce biologically relevant DNA-strand breaks in any of the exposed tissues evaluated. The result indicates no adverse effect of the bacterial strain on the DNA in cells of the small intestine or the colon.

The question that remains to be answered is, how strong and consistently the pks genes of $\mathrm{EcN}$ are being expressed. Messenger RNA has been demonstrated for most genes of the EcN pks locus apart from $c l b R$, with slightly higher transcript levels in early-stationary-phase cultures compared to late stationary phase (results not shown). This could suggest that colibactin is produced under nutrient limitation to compete with and eliminate other bacterial cells, an alternative function for colibactin that has been proposed [1]. Presumably, such transcription levels of EcN are insufficient to produce colibactin in amounts high enough to result in genotoxic effects in the assays applied here. The pks genes of $\mathrm{EcN}$ are highly conserved in other species (see Figure 1), and in other E. coli strains that were described to have genotoxic activity (results not shown), but a detailed comparison of their transcription activity has not yet been performed. Possibly, genes outside the $p k s$ locus affect the expression, production, or secretion of colibactin and these genes may be less conserved between $E$. coli strains than the pks genes are.

We conclude that E. coli strain Nissle 1917 does not have mutagenic activity in the in vivo mammalian Alkaline Comet assay using rats. These findings corroborate the in vitro findings determined by the Ames test, where neither live EcN bacteria, nor spent culture supernatant including EcN metabolites, were able to induce increased numbers of revertant colonies in different Ames test strains. We conclude that $\mathrm{EcN}$ has no mutagenic, genotoxic or DNA-damaging activity.

\section{Funding Sources}

This study was financed by Ardeypharm GmbH.

\section{Author's Contributions}

$\mathrm{SD}, \mathrm{BK}, \mathrm{MS}$, and RvB conceptualized and designed the study. All authors analyzed and interpreted data. TMW performed gene comparison and wrote the first draft. All authors edited and approved the final manuscript.

\section{Conflict of Interest}

$\mathrm{SD}, \mathrm{BK}, \mathrm{MS}$, and RvB are employed by Ardeypharm $\mathrm{GmbH}$, the producer of probiotic products containing E. coli Nissle 1917. TMW received financial support from Ardeypharm $\mathrm{GmbH}$.

Acknowledgements. We thank Dr. Sylvana Mueller at Mueller Pharma Consult for scientific input and our colleagues at Eurofins BioPharma Product Testing and at BSL Munich for experimental support.

\section{References}

1. Wassenaar TM. Insights from 100 years of studying probiotic E. coli. Eur J Microbiol Immunol. 2016;6:147-61.

2. Homburg S, Oswald E, Hacker J, Dobrindt U. Expression analysis of the colibactin gene cluster coding for a novel polyketide in Escherichia coli. FEMS Microbiol Lett. 2007;275:255-62.

3. Nougayrède JP, Homburg S, Taieb F, Boury M, Brzuszkiewicz E, Gottschalk G, et al. Escherichia coli induces DNA double-strand breaks in eukaryotic cells. Science 2006;313:848-51.

4. Cuevas-Ramos G, Petit CR, Marcq I, Boury M, Oswald E, Nougayrède JP. Escherichia coli induces DNA damage in vivo and triggers genomic instability in mammalian cells. Proc Natl Acad Sci U S A. 2010;107:11537542 .

5. Arthur JC, Perez-Chanona E, Mühlbauer M, Tomkovich S, Uronis JM, Fan TJ, et al. Intestinal inflammation targets cancer-inducing activity of the microbiota. Science. 2012;338:120-23.

6. Prorok-Hamon M, Friswell MK, Alswied A, Roberts CL, Song F, Flanagan PK, et al. Colonic mucosa-associated diffusely adherent afaC+ Escherichia coli expressing lpfA and pks are increased in inflammatory bowel disease and colon cancer. Gut. 2014;63:761-70.

7. Bonnet M, Buc E, Sauvanet P, Darcha C, Dubois D, Pereira B, et al. Colonization of the human gut by $E$. coli and colorectal cancer risk. Clin Cancer Res. 2014;20:859-86.

8. Raisch J, Buc E, Bonnet M, Sauvanet P, Vazeille E, de Vallée A, et al. Colon cancer-associated B2 Escherichia coli colonize gut mucosa and promote cell proliferation. World J Gastroenterol. 2014;20:6560-72.

9. Gagnière J, Raisch J, Veziant J, Barnich N, Bonnet R, Buc E, et al. Gut microbiota imbalance and colorectal cancer. World J Gastroenterol. 2016;22:501-18.

10. Cougnoux A, Dalmasso G, Martinez R, Buc E, Delmas J, Gibold L, et al. Bacterial genotoxin colibactin promotes colon tumor growth by inducing a senescence-associated secretory phenotype. Gut. 2014;63:1932-42.

11. Maron DE, Ames BN. Revised methods for the Salmonella mutagenicity test. Mutat Res. 1983;113:173-215.

12. Mortelsmans K, Zeiger E. The Ames Salmonella/microsome mutagenicity assay. Mutat Res. 2000;455:29-60.

13. Östling O, Johanson KJ. Microelectrophoretic study of radiationinduced DNA damages in individual mammalian cells. Biochem Biophys Res Commun. 1984;123:291-98.

14. Singh NP, McCoy MT, Tice RR, Schneider EL. A simple technique for quantitation of low levels of DNA damage in individual cells. Exp Cell Res. 1988;175:184-91.

15. Hartmann A, Agurell E, Beevers C, Brendler-Schwaab S, Burlinson B, Clay $\mathrm{P}$, et al. Recommendations for conducting the in vivo alkaline Comet assay. Mutagenesis. 2003;18:45-54.

16. Vasquez MZ. Recommendations for safety testing with the in vivo comet assay. Mutat Res. 2012;747:142-56.

17. OECD Guidelines for Testing of Chemicals, No. 471: Bacterial Reverse Mutation Test. adopted July 21, 1997. doi: 10.1787/9789264071247-en. 
18. OECD Guidelines for Testing of Chemicals, No. 489: In Vivo Mammalian Alkaline Comet Assay, adopted July 29, 2016. 10.1787/ 9789264264885-en.

19. Kang SH, Kwon JY, Lee JK, Seo YR. Recent advances in in vivo genotoxicity testing: prediction of carcinogenic potential using comet and micronucleus assay in animal models. J Cancer Prev. 2013;18:277-88.

20. Tice RR, Strauss GH. The single cell gel electrophoresis/Comet assay: a potential tool for detecting radiation-induced DNA damage in humans. Stem Cells. 1995;13(S1):207-14

21. Collins AR. The Comet assay for DNA damage and repair: principles, applications, and limitations. Mol Biotechnol. 2004;26:249-61.

22. Wassenaar TM. E. coli and colorectal cancer: a complex relationship that deserves a critical mindset. Crit Rev Microbiol. 2018;44:619-32.

23. EC Regulation No. 440/2008 B. 13/14: Mutagenicity - Reverse Mutation Test using Bacteria, May 30, 2008

24. OECD Guidelines for Testing of Chemicals, number 423: Acute Ora Toxicity - Acute Toxic Class Method, adopted December 17, 2001. 10.1787/ 9789264071001-en.

25. EPA Health Effects Test Guidelines, OPPTS 870.5100, Bacterial Reverse Mutation Test EPA 712-C-98-247, August 1998.

26. OECD Guidelines for Testing of Chemicals, number 420: Acute Oral Toxicity - Fixed Dose Procedure, adopted December 17, 2001. 10.1787 9789264070943-en.

27. OECD Guidelines for Testing of Chemicals, number 407: Repeated Dose 28-day Oral Toxicity Study in Rodents, adopted October 3, 2008.

28. Blum-Oehler G, Oswald S, Eiteljörge K, Sonnenborn U, Schulze J, Kruis W, et al. Development of strain-specific PCR reactions for the detection of the probiotic Escherichia coli strain Nissle 1917 in fecal samples. Res Microbiol. 2003;154:59-66.

29. O'Hara CM, Steigerwalt AG, Hill BC, Farmer JJ 3rd, Fanning GR, Brenner DJ. Enterobacter hormaechei, a new species of the family
Enterobacteriaceae formerly known as enteric group 75. J Clin Microbiol. 1989;27:2046-49.

30. Turkez H, Arslan ME, Ozdemir O. Genotoxicity testing: progress and prospects for the next decade. Expert Opin Drug Metab Toxicol. 2017;13:1089-98.

31. Zeller A, Pfuhler S, Albertini S, Bringezu F, Czich A, Dietz Y, et al. A critical appraisal of the sensitivity of in vivo genotoxicity assays in detecting human carcinogens. Mutagenesis. 2018;33:179-93.

32. Cañas MA, Giménez R, Fábrega MJ, Toloza L, Baldomà L, Badia J. Outer Membrane Vesicles from the Probiotic Escherichia coli Nissle 1917 and the Commensal ECOR12 Enter Intestinal Epithelial Cells via ClathrinDependent Endocytosis and Elicit Differential Effects on DNA Damage. PLoS One. 2016;11(8):e0160374.

33. Reuter C, Alzheimer M, Walles H, Oelschlaeger TA. An adherent mucus layer attenuates the genotoxic effect of colibactin. Cell Microbiol 2018;20(2). doi: 10.1111/cmi.12812.

34. Arthur JC, Gharaibeh RZ, Mühlbauer M, Perez-Chanona E, Uronis JM, McCafferty $\mathrm{J}$, et al. Microbial genomic analysis reveals the essential role of inflammation in bacteria-induced colorectal cancer. Nat Commun. 2014;5:4724. doi: 10.1038/ncomms5724.

35. Wilson MR, Jiang Y, Villalta PW, Stornetta A, Boudreau PD, Carrá A, Brennan CA, Chun E, Ngo L, Samson LD, Engelward BP, Garrett WS, Balbo $\mathrm{S}$, Balskus EP. The human gut bacterial genotoxin colibactin alkylates DNA. Science. 2019;363(6428) pii: eaar7785. doi: 10.1126/science.aar7785.

36. Dalmasso G, Cougnoux A, Delmas J, Darfeuille-Michaud A, Bonnet R. The bacterial genotoxin colibactin promotes colon tumor growth by modifying the tumor microenvironment. Gut Microbes. 2014;5:675-80

37. Li R, Helbig L, Fu J, Bian X, Herrmann J, Baumann M, Stewart AF, Müller R, Li A, Zips D, Zhang Y. Expressing cytotoxic compounds in Escherichia coli Nissle 1917 for tumor-targeting therapy. Res Microbiol. 2019;170:74-9. 